\title{
CRE Solvability, Exact Soliton-Cnoidal Wave Interaction Solutions, and Nonlocal Symmetry for the Modified Boussinesq Equation
}

\author{
Wenguang Cheng' and Biao $\mathrm{Li}^{2}$ \\ ${ }^{1}$ Department of Mathematics, Yuxi Normal University, Yuxi 653100, China \\ ${ }^{2}$ Ningbo Collaborative Innovation Center of Nonlinear Hazard System of Ocean and Atmosphere, Ningbo University, \\ Ningbo 315211, China
}

Correspondence should be addressed to Biao Li; libiao@nbu.edu.cn

Received 27 February 2016; Accepted 12 May 2016

Academic Editor: Andrei D. Mironov

Copyright (C) 2016 W. Cheng and B. Li. This is an open access article distributed under the Creative Commons Attribution License, which permits unrestricted use, distribution, and reproduction in any medium, provided the original work is properly cited.

\begin{abstract}
It is proved that the modified Boussinesq equation is consistent Riccati expansion (CRE) solvable; two types of special solitoncnoidal wave interaction solution of the equation are explicitly given, which is difficult to be found by other traditional methods. Moreover, the nonlocal symmetry related to the consistent tanh expansion (CTE) and the residual symmetry from the truncated Painlevé expansion, as well as the relationship between them, are obtained. The residual symmetry is localized after embedding the original system in an enlarged one. The symmetry group transformation of the enlarged system is derived by applying the Lie point symmetry approach.
\end{abstract}

\section{Introduction}

The exact solutions for nonlinear evolution equations (NLEEs) arising from many science fields are important because of their wide applications in explaining physical phenomena. Many powerful methods for obtaining the exact solutions of NLEEs have been presented, such as the inverse scattering transformation [1], the Darboux transformations (DT) [2], the Bäcklund transformation (BT) [3], the Lie group method $[4,5]$, Hirota's bilinear method [6], the Painlevé analysis [7], separated variable method [8], homogeneous balance method [9], and tanh function expansion method [10]. For these methods, the interaction solutions among different nonlinear excitations such as solitons on a cnoidal wave background are difficult to obtain. The soliton solutions on the background of the periodic solutions which are given by the elliptic theta function can be found with the help of Cauchy-Baker kernel [11, 12].

Recently, Lou found that the symmetry from the truncated Painlevé expansion is just the residue with respect to the singular manifold and proposed residual symmetry $[13,14]$. Furthermore, by developing the truncated Painlevé expansion, Lou $[15,16]$ established the consistent Riccati expansion (CRE) method, which is a simple but effective method to construct interaction solutions among different nonlinear excitations. The method is valid for some nonlinear models like the bosonized supersymmetric Korteweg-de Vries (KdV) model [14], the nonlinear Schrödinger system [16], the Broer-Kaup System [17], and so on [18-22].

In this paper, we focus on investigating the CRE solvability and nonlocal symmetry of the modified Boussinesq equation:

$$
3 u^{2} u_{x}+3 u_{x} \int u_{t} d x-\frac{1}{2} u_{x x x}-\frac{3}{2} \int u_{t t} d x=0
$$

which was proposed by Hirota and Satsuma [23] from a BT of the Boussinesq equation:

$$
u_{1 t t}+\left(3 u_{1}^{2}+\frac{1}{3} u_{1 x x}\right)_{x x}=0
$$


Equation (1) is called the modified Boussinesq equation since it is linked with the known Boussinesq equation (2) by the Miura transformation:

$$
u_{1}=\frac{1}{3}\left( \pm u_{x}-u^{2}-\int u_{t} d x\right)
$$

The generalized Hamiltonian form and the corresponding finite-dimensional integrable systems of (1) are obtained with the help of the nonlinearization approach of Lax pairs [24]. The Painlevé property [25] and similarity solutions $[26,27]$ have been studied. To our knowledge, the analytic interaction solutions among different nonlinear excitations for (1) have not been obtained up to now. Here we will study the interaction solution between the soliton and the cnoidal periodic wave of (1).

The paper is arranged as follows. Section 2 is devoted to the CRE method for the modified Boussinesq equation. As a result, two types of special explicit interaction solution between the soliton and the cnoidal periodic wave of this equation are given. In Section 3, for the modified Boussinesq equation, we derive the nonlocal symmetry related to the CTE and the nonlocal residual symmetry from the truncated Painlevé expansion. The relationship between them is presented. Then the corresponding symmetry group transformation is found in the process of the localization of residual symmetry. The last section is a short summary and discussion.

\section{CRE Solvability and Soliton-Cnoidal Wave Interaction Solutions}

2.1. CRE Solvability. Based on the CRE method in [15], the possible truncated expansion expression for (1) has the form

$$
u=u_{1} R(w)+u_{0}
$$

where $u_{0}, u_{1}$, and $w$ are undetermined functions of spacetime $x$ and $t$ and $R(w)$ satisfies the Riccati equation

$$
R_{w}=a_{0}+a_{1} R+a_{2} R^{2}
$$

which includes $\tanh (w)$ as a special case. Differentiating with respect to $x$, (1) becomes

$$
\begin{gathered}
12 u_{x x}^{2} u_{t}+12 u_{x}^{3} u_{x x}+36 u u_{x} u_{x x}^{2}+6 u_{x} u_{x x} u_{x t} \\
-u_{x x} u_{x x x x x}-3 u_{x x} u_{x t t}-12 u u_{x}^{2} u_{x x x} \\
-6 u_{x} u_{x x x} u_{t}+u_{x x x} u_{x x x x}+3 u_{x x x} u_{t t}=0 .
\end{gathered}
$$

Substituting (4) with (5) into (6) and vanishing all the coefficients of the powers of $R(w)$, we obtain ten overdetermined equations for only three undetermined functions. Fortunately, these overdetermined equations are consistent and possess the following solution:

$$
\begin{aligned}
& u_{1}=-a_{2} w_{x} \\
& u_{0}=-\frac{1}{2} a_{1} w_{x}-\frac{1}{2} \frac{w_{x x}}{w_{x}}-\frac{1}{2} \frac{w_{t}}{w_{x}}
\end{aligned}
$$

and the function $w$ satisfies a generalization of the Schwartzian form of (1):

$$
3 \mathscr{C}_{t}+3 \mathscr{C}_{x}+\mathcal{S}_{x}-\delta w_{x} w_{x x}=0
$$

where

$$
\begin{aligned}
& \mathscr{C}=\frac{w_{t}}{w_{x}}, \\
& \mathcal{S}=\frac{w_{x x x}}{w_{x}}-\frac{3}{2} \frac{w_{x x}^{2}}{w_{x}^{2}} \\
& \delta=a_{1}^{2}-4 a_{0} a_{2} .
\end{aligned}
$$

Due to consistency of the overdetermined system, we call the modified Boussinesq equation CRE solvable.

2.2. CTE Solvability and Soliton-Cnoidal Wave Interaction Solutions. We consider the function $R(w)$ in Riccati equation (5) as the following special solution:

$$
R(w)=\tanh (w)
$$

which means that a CRE solvable system must be consistent tanh expansion (CTE) solvable, and vice versa. Meanwhile, truncated expansion expression (4) is changed as

$$
u=u_{0}+u_{1} \tanh (w)
$$

where $u_{0}, u_{1}$, and $w$ are undetermined by (7) and (8) with $a_{0}=1, a_{1}=0, a_{2}=-1$, and $\delta=4$. Thus, we have

$$
\begin{aligned}
& u_{1}=w_{x}, \\
& u_{0}=-\frac{1}{2} \frac{w_{x x}}{w_{x}}-\frac{1}{2} \frac{w_{t}}{w_{x}}
\end{aligned}
$$

and the function $w$ satisfies

$$
3 \mathscr{C}_{t}+3 \mathscr{C}_{x}+\mathcal{S}_{x}-4 w_{x} w_{x x}=0 .
$$

In brief, we arrive at the following nonauto-BT theorem for (1).

Theorem 1. If $w$ is a solution of (13), then $u$, with

$$
u=w_{x} \tanh (w)-\frac{1}{2} \frac{w_{x x}}{w_{x}}-\frac{1}{2} \frac{w_{t}}{w_{x}},
$$

is a solution of modified Boussinesq equation (1).

Theorem 1 shows that the single soliton solution of (1) is only the straight line solution $w=k_{0} x+\omega_{0} t$ of (13); the interaction solutions between solitons and other nonlinear excitations of (1) can be constructed by solving (13). To find the interaction solutions between one soliton and other nonlinear waves of (1), we consider $w$ in the form

$$
w=k_{0} x+\omega_{0} t+g
$$


where $g$ is a function with respect to $x$ and $t$. In this study, we only discuss the solutions with the form

$$
\begin{aligned}
& w=k_{0} x+\omega_{0} t+W(X), \\
& X=k_{1} x+\omega_{1} t .
\end{aligned}
$$

Substituting (16) into (13), we can find that $W_{1}(X)$ satisfies

$$
\begin{aligned}
W_{1}(X)_{X}^{2}= & 4 W_{1}(X)^{4}+2 a_{1} W_{1}(X)^{3}+2 a_{2} W_{1}(X)^{2} \\
& +2 a_{3} W_{1}(X)+a_{4}
\end{aligned}
$$

with

$$
\begin{aligned}
& W(X)_{X}=W_{1}(X), \\
& a_{1}=\frac{4 k_{0}-C_{1} k_{1}^{3}}{k_{1}}, \\
& a_{2}=\frac{2 k_{0}^{2}-3 C_{1} k_{0} k_{1}^{3}+C_{2} k_{1}^{3}}{k_{1}^{2}}, \\
& a_{3}=\frac{-3 k_{0} \omega_{1}^{2}+3 k_{1} \omega_{0} \omega_{1}-3 C_{1} k_{0}^{2} k_{1}^{5}+2 C_{2} k_{0} k_{1}^{5}}{k_{1}^{5}},
\end{aligned}
$$

$a_{4}$

$$
=\frac{4 k_{0} k_{1} \omega_{0} \omega_{1}-5 k_{0}^{2} \omega_{1}^{2}+k_{1}^{2} \omega_{0}^{2}-2 C_{1} k_{0}^{3} k_{1}^{5}+2 C_{2} k_{0}^{2} k_{1}^{5}}{k_{1}^{6}},
$$

and $C_{1}, C_{2}$ are arbitrary constants. Then (1) has the explicit solution expressed as

$$
\begin{gathered}
u=\left(k_{0}+k_{1} W_{1}(X)\right) \tanh \left(k_{0} x+\omega_{0} t+W(X)\right) \\
-\frac{\omega_{0}+\omega_{1} W_{1}(X)+k_{1}^{2} W_{1}(X)_{X}}{2\left(k_{0}+k_{1} W_{1}(X)\right)} .
\end{gathered}
$$

It is clear that (17) has abundant explicit solutions in terms of Jacobi elliptic functions. Hence, solution (19) exhibits the interactions between one soliton and cnoidal periodic waves. In what follows, only two nontrivial cases are considered in detail to obtain this kind of solution.

Case 1. A simple solution of (17) is given as

$$
W(X)=c E_{\pi}(\operatorname{sn}(X, m), n, m),
$$

which leads to the soliton-cnoidal wave interaction solution of (1) as follows:

$$
\begin{aligned}
u= & \left(k_{0}-\frac{c k_{1}}{n S^{2}-1}\right) \tanh \left(k_{0} x+\omega_{0} t+c E_{\pi}(S, n, m)\right) \\
& +\frac{c k_{1}^{2} n S C D}{\left(n S^{2}-1\right)\left[c k_{1}-k_{0}\left(n S^{2}-1\right)\right]} \\
& -\frac{c \omega_{1}-\omega_{0}\left(n S^{2}-1\right)}{2\left[c k_{1}-k_{0}\left(n S^{2}-1\right)\right]},
\end{aligned}
$$

where $\left\{k_{0}, k_{1}, m, n\right\}$ are arbitrary constant, $S \equiv \operatorname{sn}\left(k_{1} x+\right.$ $\left.\omega_{1} t, m\right), C \equiv \operatorname{cn}\left(k_{1} x+\omega_{1} t, m\right), D \equiv \operatorname{dn}\left(k_{1} x+\omega_{1} t, m\right)$, and

$$
\begin{aligned}
& c^{2}=-\frac{(n-1)\left(m^{2}-n\right)}{n}, \\
& \omega_{1}=-\frac{4 c k_{0}^{3} k_{1} n+c^{2} k_{1}^{4} m^{2}+2 c k_{0} k_{1}^{3}\left(3 m^{2}-m^{2} n-n\right)+3 k_{0}^{2} k_{1}^{2}\left(3 m^{2}-2 m^{2} n+n^{2}-2 n\right)}{3 \sqrt{c k_{0} n\left[c k_{0}^{3} n+c^{2} k_{1}^{3} m^{2}+c k_{0} k_{1}^{2}\left(3 m^{2}-m^{2} n-n\right)+k_{0}^{2} k_{1}\left(3 m^{2}-2 m^{2} n+n^{2}-2 n\right)\right]}} \\
& \omega_{0}=-\frac{2 k_{1}^{2} m^{2}\left(3 k_{0}+c k_{1}\right)\left(3 k_{0}-2 k_{0} n+c k_{1}\right)}{3 c \omega_{1} n}+\frac{k_{0}\left(4 c k_{1}^{4}+3 c \omega_{1}^{2}+12 k_{0} k_{1}^{3}-6 k_{0} k_{1}^{3} n-8 c k_{0}^{2} k_{1}^{2}\right)}{3 c k_{1} \omega_{1}} .
\end{aligned}
$$

In solution (21), $E_{\pi}(\zeta, n, m)$ is the third type of incomplete elliptic integral.

The dynamic behavior of soliton-cnoidal wave interaction solution (21) with the parameters $k_{0}=1, k_{1}=1.2$, $m=0.9$, and $n=0.5$ is illustrated in Figure 1. It can be seen from Figure 1 that a soliton moves on a cnoidal wave background instead of moving on the plane continuous wave background. This kind of solution can be applicable to describe some interesting physical phenomena, such as the Fermionic quantum plasma [28].

Case 2. Another special solution of (17) reads as follows:

$$
W(X)=A \operatorname{arctanh}(\operatorname{sn}(X, m))
$$

which yields the soliton-cnoidal wave interaction solution of (1):

$$
\begin{aligned}
u= & \left(k_{0}-\frac{k_{1} C D}{2\left(S^{2}-1\right)}\right) \\
& \cdot \tanh \left(k_{0} x+\omega_{0} t+\frac{1}{2} \operatorname{arctanh}(S)\right) \\
& +\frac{k_{1}^{2} S C^{2} D^{2}}{\left(S^{2}-1\right)\left[k_{1} C D-2 k_{0}\left(S^{2}-1\right)\right]} \\
& +\frac{k_{1}^{2} S\left(m^{2} C^{2}+D^{2}\right)+2 \omega_{0}\left(S^{2}-1\right)-\omega_{1} C D}{2\left[k_{1} C D-2 k_{0}\left(S^{2}-1\right)\right]}
\end{aligned}
$$




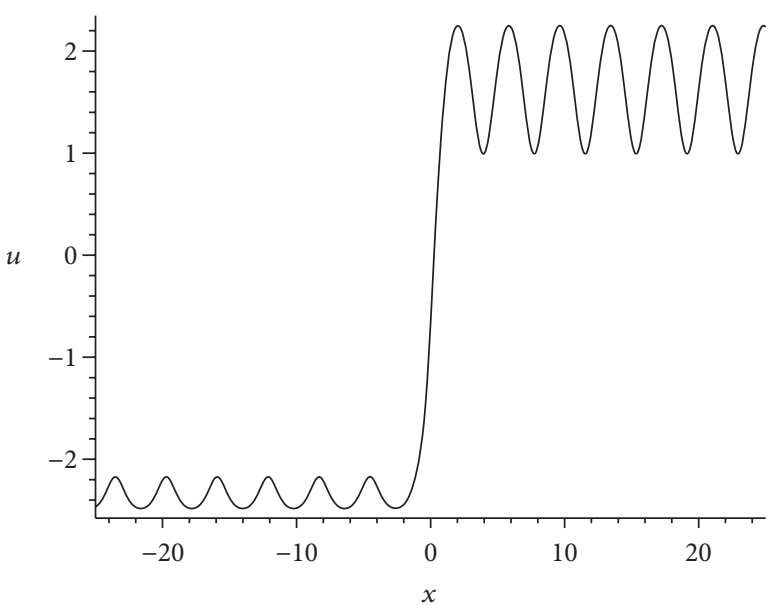

(a)

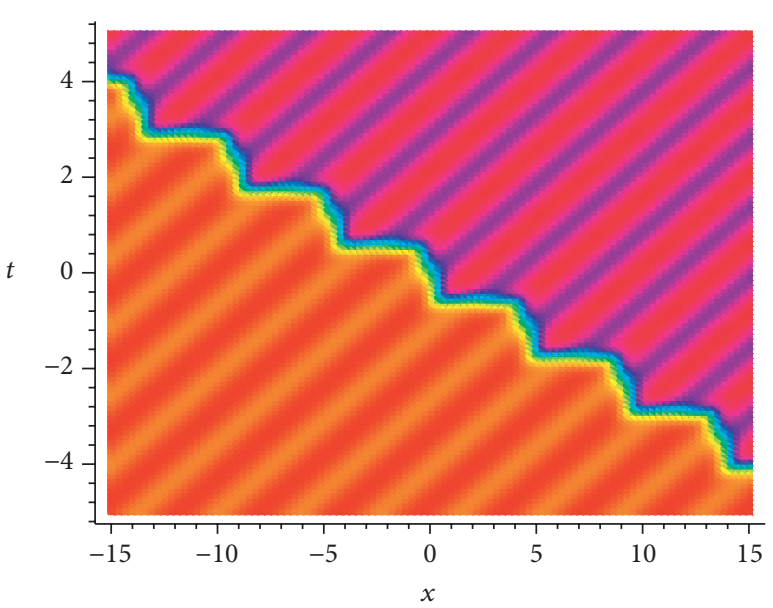

(b)

FIGURE 1: Interaction between the soliton and the cnoidal periodic wave via solution (21) with the parameters $k_{0}=1, k_{1}=1.2, m=0.9$, and $n=0.5$. (a) The profile of the special structure at $t=0$. (b) Overhead view of the wave.

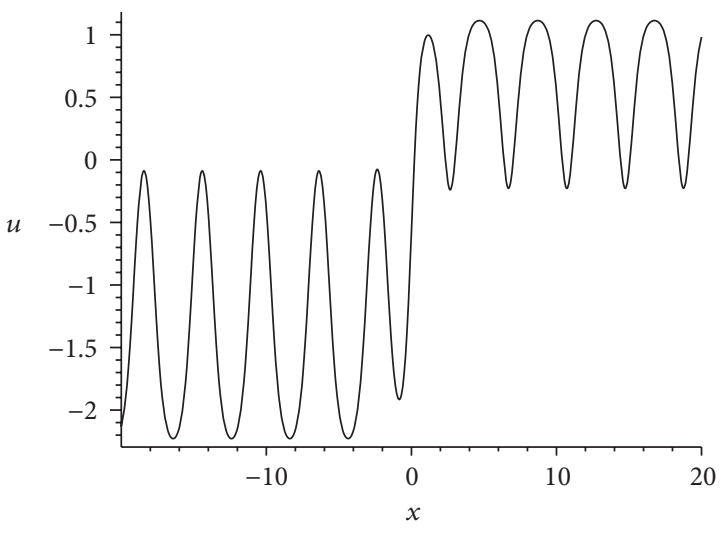

(a)

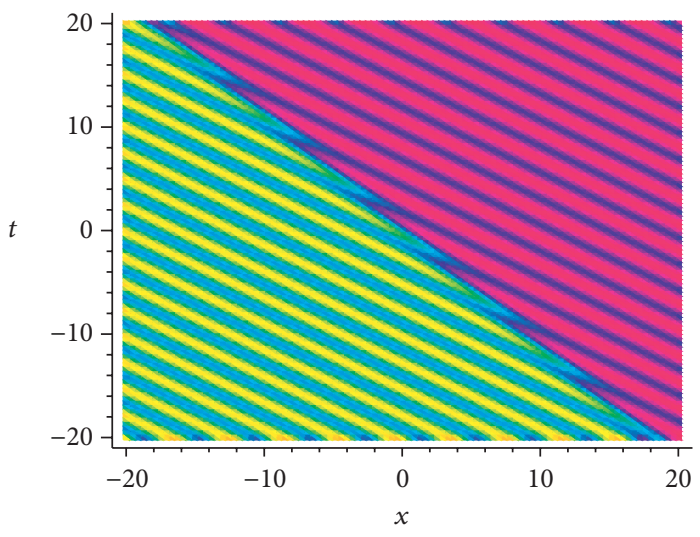

(b)

FiguRE 2: Soliton-cnoidal wave interaction solution for (1) expressed by (24) with the parameters selected as $k_{0}=1, k_{1}=1.2$, and $m=1.5$. (a) One-dimensional image at $t=0$. (b) Overhead view of the wave.

where $\left\{k_{0}, k_{1}, m\right\}$ are arbitrary constant, $S \equiv \operatorname{sn}\left(k_{1} x+\omega_{1} t, m\right)$, $C \equiv \operatorname{cn}\left(k_{1} x+\omega_{1} t, m\right), D \equiv \operatorname{dn}\left(k_{1} x+\omega_{1} t, m\right)$, and

$$
\begin{aligned}
& A=\frac{1}{2}, \\
& \omega_{0} \\
& =-\frac{8 k_{0}^{2}\left(2 k_{0}^{2}-k_{1}^{2}\right)-k_{1}^{2}\left(8 k_{0}^{2}-3 k_{1}^{2}\right)}{6 \sqrt{\left(2 k_{0}-k_{1}\right)\left(2 k_{0}+k_{1}\right)\left(2 k_{0}-k_{1} m\right)\left(2 k_{0}+k_{1} m\right)}},
\end{aligned}
$$

$\omega_{1}$

$$
=\frac{2 k_{0} k_{1}\left(8 k_{0}^{2}-k_{1}^{2}-k_{1}^{2} m^{2}\right)}{3 \sqrt{\left(2 k_{0}-k_{1}\right)\left(2 k_{0}+k_{1}\right)\left(2 k_{0}-k_{1} m\right)\left(2 k_{0}+k_{1} m\right)}} .
$$

In Figure 2, we plot soliton-cnoidal wave interaction solution (24) with the parameters selected as $k_{0}=1, k_{1}=$ 1.2 , and $m=1.5$. It presents that a soliton propagates on a cnoidal wave background instead of propagating on the plane continuous wave background.

\section{Nonlocal Symmetry and Its Localization}

3.1. Nonlocal Symmetry. To derive the nonlocal symmetry related to the CTE, we give a nonauto-BT theorem for modified Boussinesq equation (1) as follows.

Theorem 2. If $w$ is a solution of (13), then $u$, with

$$
u=-w_{x}-\frac{1}{2} \frac{w_{x x}}{w_{x}}-\frac{1}{2} \frac{w_{t}}{w_{x}}
$$

is a solution of modified Boussinesq equation (1).

Proof. By direct substitution, a symmetry $\sigma^{u}$ of (6) is defined as a solution of its linearized equation, which means 
(6) is form invariant under the infinitesimal transformation

$$
u \longrightarrow u+\epsilon \sigma^{u}
$$

with the infinitesimal parameter $\epsilon$.

Proposition 3. Modified Boussinesq equation (1) has a nonlocal symmetry given by

$$
\sigma^{u}=w_{x} e^{2 w}
$$

where $w$ satisfies (13).

Proof. By direct calculation, we substitute (28) into the linearized equation of (6) with the help of the nonauto-BT (26) in Theorem 2 and the $w$ equation (13).

Now, applying the following transformation:

$$
\phi=\frac{1}{1-\tanh (w)},
$$

to the nonlocal symmetry (28), we find

$$
\sigma^{u}=\phi_{x}
$$

which is the residual symmetry of (1).

Here we can obtain residual symmetry (30) from the truncated Painlevé expansion. Balancing the nonlinear and dispersive terms in (1), the truncated Painlevé expansion can be written as

$$
u=\frac{u_{0}}{\phi}+u_{1}
$$

where $\phi$ is the singular manifold and $u_{0}$ and $u_{1}$ are functions of $(x, t)$ to be determined later.

Substituting (31) into (6) and vanishing coefficients of the each powers of $1 / \phi$, we obtain

$$
\begin{aligned}
& u_{0}=\phi_{x} \\
& u_{1}=-\frac{1}{2} \frac{\phi_{x x}}{\phi_{x}}-\frac{1}{2} \frac{\phi_{t}}{\phi_{x}}
\end{aligned}
$$

Consequently,

$$
u=\frac{\phi_{x}}{\phi}-\frac{1}{2} \frac{\phi_{x x}}{\phi_{x}}-\frac{1}{2} \frac{\phi_{t}}{\phi_{x}}
$$

is a solution of (1) with $\phi$ satisfying Schwartzian form of (1):

$$
3 C_{t}+3 C C_{x}+S_{x}=0
$$

where the notations $C$ and $S$ are defined as

$$
\begin{aligned}
& C=\frac{\phi_{t}}{\phi_{x}}, \\
& S=\frac{\phi_{x x x}}{\phi_{x}}-\frac{3}{2} \frac{\phi_{x x}^{2}}{\phi_{x}^{2}} .
\end{aligned}
$$

Schwarzian equation (34) is invariant under the Möbious transformation

$$
\phi \longrightarrow \frac{a+b \phi}{c+d \phi}, \quad(a d \neq b c),
$$

which means (34) possesses the following Lie point symmetries in the form of

$$
\sigma^{\phi}=a_{1}+b_{1} \phi+c_{1} \phi^{2}
$$

with $a_{1}, b_{1}$, and $c_{1}$ being arbitrary constants. From the above standard truncated Painlevé expansion, we have the BT theorem as follow.

Theorem 4. If $\phi$ is a solution of the Schwartzian equation (34), then

$$
u=-\frac{1}{2} \frac{\phi_{x x}}{\phi_{x}}-\frac{1}{2} \frac{\phi_{t}}{\phi_{x}}
$$

is a nonauto-BT between $\phi$ and the solution $u$ of modified Boussinesq equation (1).

The residual of truncated Painlevé expansion (31) with the singular manifold $\phi$, that is, $u_{0}$, is a symmetry of (1) with the solution $u_{1}$. Thus, (30) is the residual symmetry of (1). It should be emphasized that the above residual symmetry is just related to the Möbious transformation symmetry (37) by the linearized equation of nonauto-BT (38) in Theorem 4.

3.2. Localization of Residual Symmetry. To find out the symmetry group transformation of nonlocal residual symmetry, we should solve the following initial value problem:

$$
\begin{aligned}
\frac{d \widehat{u}(\varepsilon)}{d \varepsilon} & =\widehat{\phi_{x}}(\varepsilon), \\
\widehat{u}(0) & =u,
\end{aligned}
$$

where $\varepsilon$ is an arbitrary group parameter.

Due to the intrusion of the function $\widehat{\phi}(\varepsilon)$ and its differentiations, it is difficult to solve the above initial value problem according to the Lie first theorem. Thus, one may extend the original system such that nonlocal residual symmetry becomes the local Lie point symmetry of a closed prolonged system.

The nonlocal residual symmetry of (1) can be localized to the Lie point symmetry

$$
\begin{aligned}
\sigma^{u} & =g, \\
\sigma^{g} & =-2 \phi g, \\
\sigma^{\phi} & =-\phi^{2},
\end{aligned}
$$

for the prolonged system

$$
\begin{aligned}
& 3 u^{2} u_{x}+3 u_{x} \int u_{t} d x-\frac{1}{2} u_{x x x}-\frac{3}{2} \int u_{t t} d x=0, \\
& u=-\frac{1}{2} \frac{\phi_{x x}}{\phi_{x}}-\frac{1}{2} \frac{\phi_{t}}{\phi_{x}} \\
& g=\phi_{x} .
\end{aligned}
$$


Prolonged system (41) is closed after covering dependent variables $u, g$, and $\phi$ with the vector form

$$
V=g \frac{\partial}{\partial u}-2 \phi g \frac{\partial}{\partial g}-\phi^{2} \frac{\partial}{\partial \phi}
$$

Correspondingly, initial value problem (39) is changed as

$$
\begin{aligned}
& \frac{d \widehat{u}(\varepsilon)}{d \varepsilon}=\widehat{g}(\varepsilon), \quad \widehat{u}(0)=u, \\
& \frac{d \widehat{g}(\varepsilon)}{d \varepsilon}=-2 \widehat{\phi}(\varepsilon) \widehat{g}(\varepsilon), \quad \widehat{g}(0)=g, \\
& \frac{d \widehat{\phi}(\varepsilon)}{d \varepsilon}=-\widehat{\phi}(\varepsilon)^{2}, \quad \widehat{\phi}(0)=\phi .
\end{aligned}
$$

By solving the above initial value problem, we arrive at the symmetry group transformation theorem as follow.

Theorem 5. If $\{u, g, \phi\}$ is a solution of prolonged system (41), then so is $\{\widehat{u}, \widehat{g}, \widehat{\phi}\}$ with

$$
\begin{aligned}
& \widehat{u}(\varepsilon)=u+\frac{g \varepsilon}{1+\phi \varepsilon}, \\
& \widehat{g}(\varepsilon)=\frac{g}{(1+\phi \varepsilon)^{2}}, \\
& \widehat{\phi}(\varepsilon)=\frac{\phi}{1+\phi \varepsilon} .
\end{aligned}
$$

Finite transformation (44) provides a way to generate a new solution from old one. It is necessary to point out that the nonlocal residual symmetry is nothing but the infinitesimal form of the finite transformation. Actually, if we set

$$
\begin{aligned}
1+\phi \varepsilon & =\phi, \\
g \varepsilon & =\phi_{x},
\end{aligned}
$$

the first equation of (44) is just the truncated Painlevé expansion (31) with (32).

\section{Summary and Discussion}

In conclusion, the modified Boussinesq equation is proved to be CRE integrable. The CTE method which is a special simplified form leads to a nonauto-BT, which strengthens a single soliton to a straight line solution. Two types of special interaction solution between the soliton and the cnoidal periodic wave are derived by means of the nonauto-BT.

On the other hand, for the modified Boussinesq equation, the nonlocal symmetry related to the CTE is derived. Under the transformation $\phi=1 /(1-\tanh (w))$, this nonlocal symmetry is changed as the residual symmetry which can be obtained from the truncated Painlevé expansion. To solve the initial value problem related to the residual symmetry, we extend the original system such that residual symmetry becomes the local Lie point symmetry of a closed prolonged system. The symmetry group transformation of the prolonged system is derived by using the Lie first theorem.

More about further integrable properties from CRE will be investigated in detail in our future research work.

\section{Competing Interests}

The authors declare that there are no competing interests regarding the publication of this paper.

\section{Acknowledgments}

The authors are grateful to Professor Y. Chen for his helpful suggestions and fruitful discussion. This work is supported by National Natural Science Foundation of China under Grant nos. 11271211, 11435005, and 11505090, K. C. Wong Magna Fund in Ningbo University, and Research Award Foundation for Outstanding Young Scientists of Shandong Province (no. BS2015SF009).

\section{References}

[1] C. S. Gardner, J. M. Greene, M. D. Kruskal, and R. M. Miura, "Method for solving the Korteweg-deVries equation," Physical Review Letters, vol. 19, no. 19, pp. 1095-1097, 1967.

[2] V. B. Matveev and M. A. Salle, Darboux Transformations and Solitons, Springer, Berlin, Germany, 1991.

[3] R. M. Miura, Bäcklund Transformation, Springer, Berlin, Germany, 1978.

[4] P. J. Olver, Applications of Lie Groups to Differential Equations, vol. 107 of Graduate Texts in Mathematics, Springer, Berlin, Germany, 1986.

[5] G. W. Bluman and S. Kumei, Symmetries and Differential Equations, Springer, New York, NY, USA, 1989.

[6] R. Hirota, "Exact solution of the Korteweg-de Vries equation for multiple collisions of solitons," Physical Review Letters, vol. 27, no. 18, pp. 1192-1194, 1971.

[7] J. Weiss, M. Tabor, and G. Carnevale, “The Painlevé property for partial differential equations," Journal of Mathematical Physics, vol. 24, no. 3, pp. 522-526, 1983.

[8] X.-Y. Tang, S.-Y. Lou, and Y. Zhang, "Localized excitations in $(2+1)$-dimensional systems," Physical Review E, vol. 66, no. 4, Article ID 046601, 17 pages, 2002.

[9] M. Wang, Y. Zhou, and Z. Li, "Application of a homogeneous balance method to exact solutions of nonlinear equations in mathematical physics," Physics Letters, Section A: General, Atomic and Solid State Physics, vol. 216, no. 1-5, pp. 67-75, 1996.

[10] A.-M. Wazwaz, "The tanh method for traveling wave solutions of nonlinear equations," Applied Mathematics and Computation, vol. 154, no. 3, pp. 713-723, 2004.

[11] P. G. Grinevich and A. Y. Orlov, "Variations of the complex structure of Riemann surfaces by vector fields on a contour and objects of the KP theory. The Krichever-Novikov problem of the action on the Baker-Akhieser functions," Functional Analysis and Its Applications, vol. 24, no. 1, pp. 61-63, 1990.

[12] P. G. Grinevich and A. Yu. Orlov, "Flag spaces in KP Theory andVirasoro action on det $D_{j}$ and Segal-Wilson $\tau$-function," http://arxiv.org/abs/math-ph/9804019vl.

[13] S. Y. Lou, "Residual symmetries and Bäcklundtransformations," http://arxiv.org/abs/1308.1140vl.

[14] X. N. Gao, S. Y. Lou, and X. Y. Tang, "Bosonization, singularity analysis, nonlocal symmetry reductions and exact solutions of supersymmetric KdV equation," Journal of High Energy Physics, vol. 2013, article 29, 2013. 
[15] S. Y. Lou, "Consistent Riccati expansion for integrable systems," Studies in Applied Mathematics, vol. 134, no. 3, pp. 372-402, 2015.

[16] S.-Y. Lou, X.-P. Cheng, and X.-Y. Tang, "Dressed dark solitons of the defocusing nonlinear Schrödinger equation," Chinese Physics Letters, vol. 31, no. 7, Article ID 070201, 2014.

[17] C.-L. Chen and S.-Y. Lou, "CTE solvability and exact solution to the Broer-Kaup system," Chinese Physics Letters, vol. 30, no. 11, Article ID 110202, 2013.

[18] D. Yang, S.-Y. Lou, and W.-F. Yu, "Interactions between solitons and cnoidal periodic waves of the Boussinesq equation," Communications in Theoretical Physics, vol. 60, no. 4, pp. 387-390, 2013.

[19] W.-G. Cheng, B. Li, and Y. Chen, "Construction of solitoncnoidal wave interaction solution for the $(2+1)$-dimensional breaking soliton equation," Communications in Theoretical Physics, vol. 63, no. 5, pp. 549-553, 2015.

[20] Y.-H. Wang and H. Wang, "Symmetry analysis and CTE solvability for the $(2+1)$-dimensional Boiti-Leon-Pempinelli equation," Physica Scripta, vol. 89, no. 12, Article ID 125203, 2014.

[21] B. Ren, "Interaction solutions for mKP equation with nonlocal symmetry reductions and CTE method," Physica Scripta, vol. 90, no. 6, Article ID 065206, 2015.

[22] X. R. Hu and Y. Q. Li, "Nonlocal symmetry and soliton-cnoidal wave solutions of the Bogoyavlenskii coupled KdV system," Applied Mathematics Letters, vol. 51, pp. 20-26, 2016.

[23] R. Hirota and J. Satsuma, "Nonlinear evolution equations generated from the Bäcklund transformation for the Boussinesq equation," Progress of Theoretical Physics, vol. 57, no. 3, pp. 797$807,1977$.

[24] H.-H. Dai and X. Geng, "Finite-dimensional integrable systems through the decomposition of a modified Boussinesq equation," Physics Letters A, vol. 317, no. 5-6, pp. 389-400, 2003.

[25] P. A. Clarkson, "The Painlevé property, a modified Boussinesq equation and a modified Kadomtsev-PETviashvili equation," Physica D, vol. 19, no. 3, pp. 447-450, 1986.

[26] G. R. W. Quispel, F. W. Nijhoff, and H. W. Capel, "Linearization of the Boussinesq equation and the modified Boussinesq equation," Physics Letters A, vol. 91, no. 4, pp. 143-145, 1982.

[27] P. A. Clarkson, "New similarity solutions for the modified Boussinesq equation," Journal of Physics A: Mathematical and General, vol. 22, no. 13, pp. 2355-2367, 1989.

[28] A. J. Keane, A. Mushtaq, and M. S. Wheatland, "Alfvén solitons in a Fermionic quantum plasma," Physical Review E, vol. 83, no. 6, Article ID 066407, 2011. 


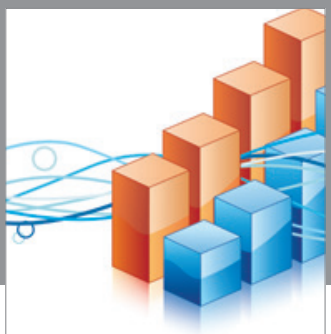

Advances in

Operations Research

vatem alat4

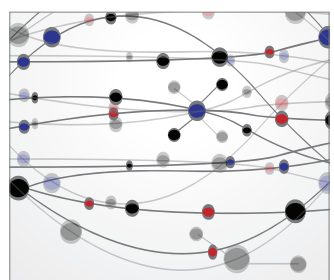

\section{The Scientific} World Journal
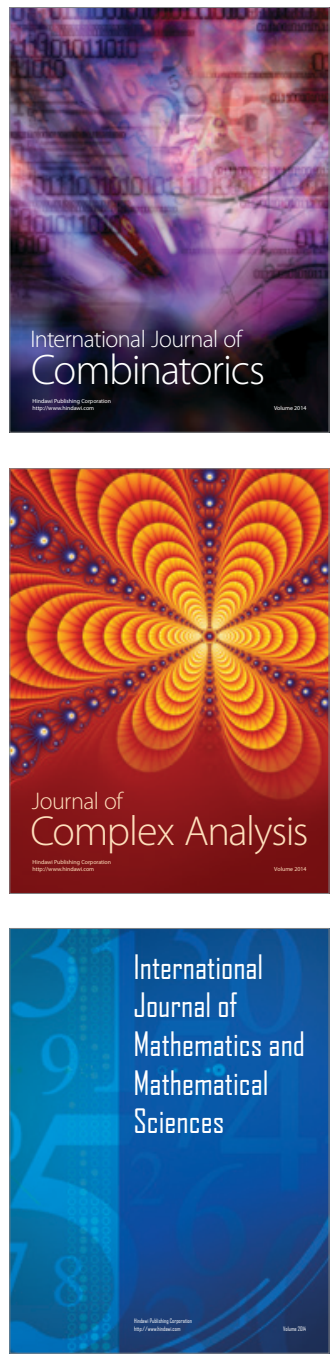
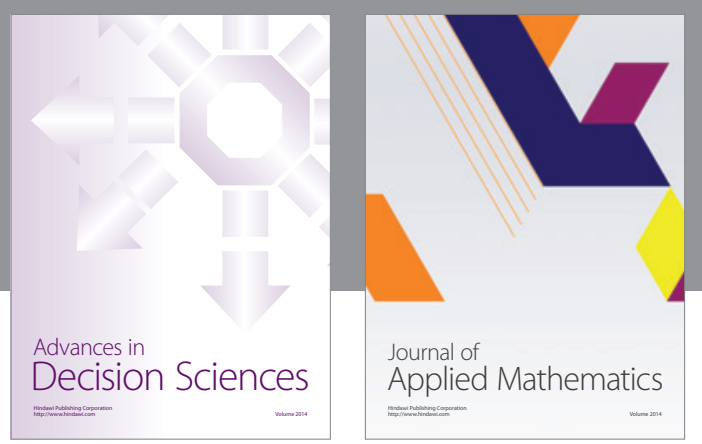

Algebra

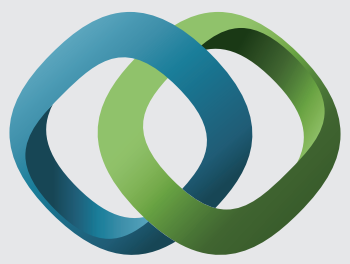

\section{Hindawi}

Submit your manuscripts at

http://www.hindawi.com
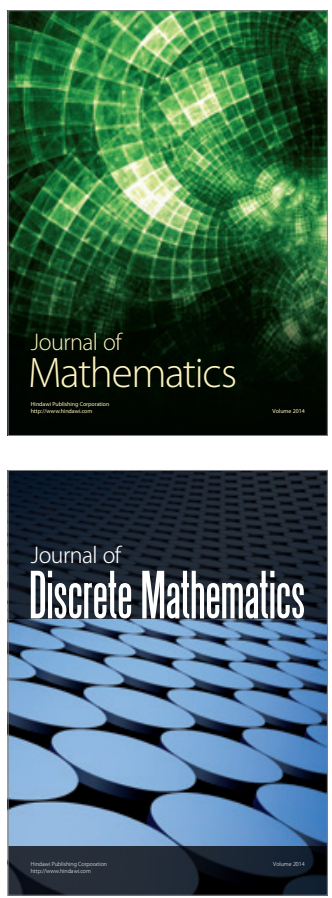

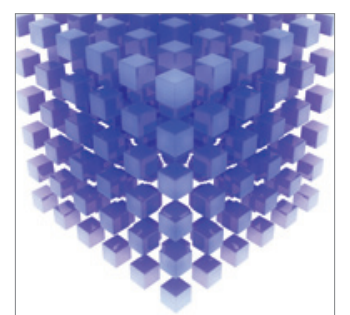

Mathematical Problems in Engineering
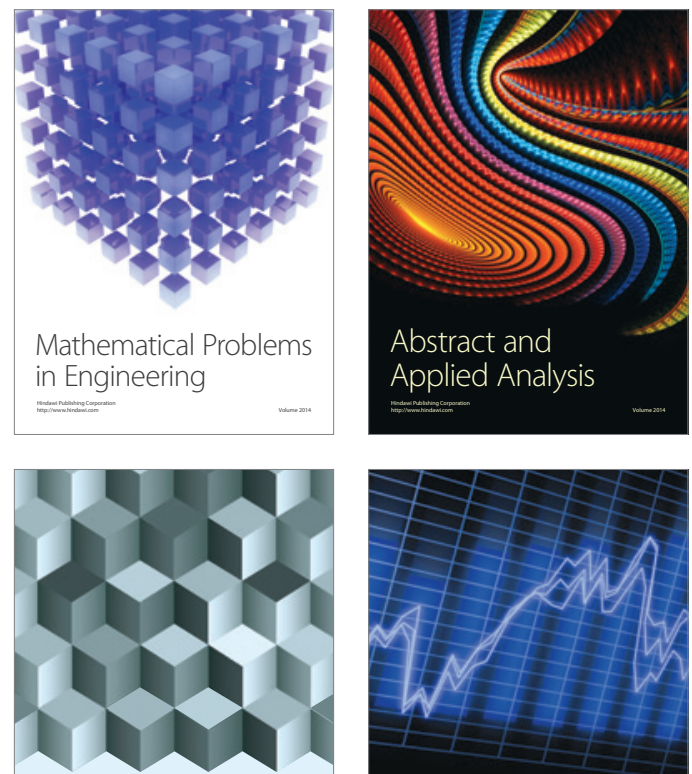

Journal of

Function Spaces

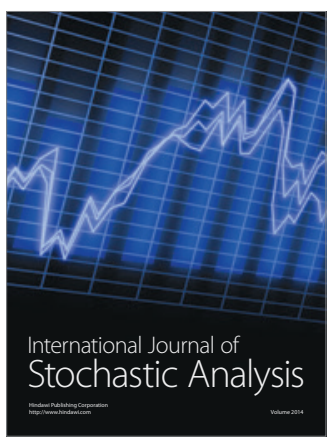

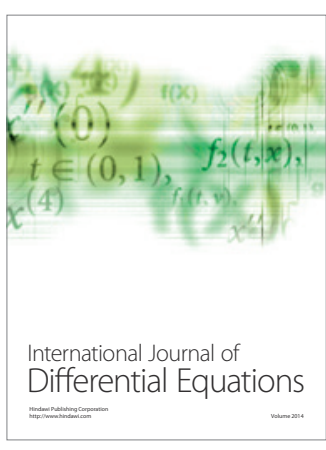
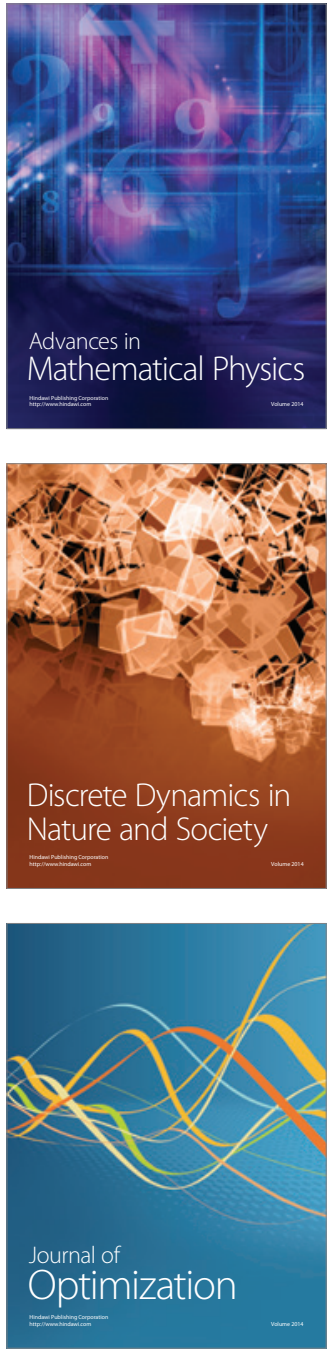